\title{
Association of Haemodynamic Indices of Central and Peripheral Pressure with Subclinical Target Organ Damage
}

\author{
Junli Zuo ${ }^{a, b}$ Shaoli Chu ${ }^{a}$ Isabella Tan ${ }^{c}$ Mark Butlin $^{c}$ Jiehui Zhao ${ }^{d}$ \\ Alberto Avolio ${ }^{c}$ \\ Departments of a Hypertension and ${ }^{b}$ Geriatrics, Ruijin Hospital North, Shanghai Jiaotong \\ University School of Medicine, Shanghai, PR China; ' $D$ Department of Biomedical Sciences, \\ Faculty of Medicine and Health Sciences, Macquarie University, Sydney, NSW, Australia; \\ dThe Department of Geriatric Nursing Hospital, Baohua, Shanghai, PR China
}

\section{Keywords}

Target organ damage - Carotid-femoral pulse wave velocity · Pulse wave analysis . Intima-media thickness · Albumin-to-creatinine ratio

\begin{abstract}
Background: Central aortic pressure has often been shown to be more closely associated with markers of vascular function and incidence of cardiovascular events compared to peripheral pressure. However, the potential clinical use of central aortic or peripheral haemodynamic indices as markers of target organ damage (TOD) has not been fully established. Methods: We evaluated associations of TOD with central aortic and peripheral haemodynamic indices (central aortic [cPP] and peripheral pulse pressure [pPP], central aortic augmentation index, and central and peripheral waveform factor) in 770 hospital inpatients (age $60 \pm 10$ years, 473 males) with primary hypertension. TOD was quantified in terms of arterial stiffness as measured by carotid-femoral pulse wave velocity (cfPWV), carotid intima-media thickness (IMT), and urine albumin-to-creatinine ratio (ACR). Subclinical TOD was defined as carotid IMT $>0.9$ $\mathrm{mm}$, urine $A C R>3.5 \mathrm{mg} / \mathrm{mmol}$ in females and $>2.5 \mathrm{mg} / \mathrm{mmol}$ in males and/or cfPWV $>12 \mathrm{~m} / \mathrm{s}$. Results: Both CPP and PPP showed significant correlation with cfPWV $(r=0.41$ vs. $0.40 ; p<$ 0.01 ), ACR ( $r=0.24$ vs. $0.27 ; p<0.01$ ) and carotid IMT ( $r=0.14$ vs. $0.15 ; p<0.01$ ). Each SD increase in pPP and cPP was associated with increased risk of cfPWV $>12 \mathrm{~m} / \mathrm{s}$ (odds ratio $[O R]=2.7$ and 2.9 for pPP and $C P P$, respectively), $A C R>2.5 \mathrm{mg} / \mathrm{mmol}(\mathrm{OR}=1.2$ and 1.4 , respectively), and carotid IMT $>0.9 \mathrm{~mm}(\mathrm{OR}=1.46$ and 1.53 , respectively). Compared to pPP, CPP had higher predictive power for TOD for age $\geq 60$ years $(\mathrm{OR}=3.07, p<0.001)$. Conclu-
\end{abstract}


Zuo et al.: Association of Haemodynamic Indices of Central and Peripheral Pressure with Subclinical Target Organ Damage

sions: Although both PPP and CPP show an association with TOD in a hypertensive population, CPP provides additional information beyond PPP associated with TOD in a hypertensive cohort. Central aortic haemodynamic indices as potential biomarkers of subclinical TOD need to be validated by further prospective studies.

(C) 2017 S. Karger AG, Basel

\section{Background}

Elevated arterial blood pressure is a significant cardiovascular risk factor due to the association with target organ damage (TOD) [1]. Recent advances in blood pressure measurement have enabled the combination of the conventional brachial sphygmomanometer with a peripheral pulse waveform to estimate central aortic pressure (AP) non-invasively [2]. A number of studies have shown that central aortic blood pressure could better reflect the load on the heart and central vasculature and organs $[3,4]$. Central aortic pulse pressure (PP) showed higher correlation with carotid atherosclerosis and the incidence of cardiovascular events than peripheral PP [5-8]. Non-invasive tonometry techniques have been developed over the past decade to enable physicians to evaluate central, aortic blood pressure. However, not all studies [9] showed that haemodynamic indices of central and peripheral pressure could be used in clinical practice as a marker of damage to regions of low blood flow resistance, such as renal abnormalities and increased carotid intima-media thickness (IMT). Carotid-femoral pulse wave velocity (cfPWV) is considered as a gold-standard measure of aortic stiffness and is a cumulative measure of the damaging effects of cardiovascular risk factors on the arterial wall with aging, especially in persons over 40 years of age [10]. We have previously shown that cfPWV itself can be considered as an index of TOD [11]. Therefore, the purpose of this study was to evaluate the potential predictive role of non-invasive haemodynamic indices of central and peripheral pressure for TOD, quantified in terms of arterial stiffness (cfPWV), carotid IMT, and renal abnormalities assessed by the urine albumin-tocreatinine ratio (ACR), in a group of essential hypertensive patients.

\section{Methods}

Study Population

The population of this cross-sectional observational study included 770 (473 males, age $60.0 \pm 10.0$ years) inpatients with primary hypertension because of presence of several cardiovascular risk factors or complications involving clinical TOD in the Department of Hypertension, Ruijin Hospital, Shanghai, China. The initial cohort was selected between July 2007 and October 2008. Inclusion criteria included 3 consecutive measurements of office systolic blood pressure (SBP) $\geq 140 \mathrm{~mm} \mathrm{Hg}$ or diastolic blood pressure (DBP) $\geq 90 \mathrm{~mm} \mathrm{Hg}$ after resting in a supine position for $5 \mathrm{~min}$, or the use of antihypertensive drugs in the absence of secondary forms of hypertension. Exclusion criteria were clinical or laboratory evidence of acute cardiocerebrovascular disease within the previous 3 months, any life-threatening disease, any condition preventing the technical quality of arterial stiffness monitoring, such as subclavian or brachial artery stenosis, or any arrhythmias.

All patients underwent a standardized questionnaire for collection of information on medical history, smoking habits, alcohol consumption and the use of medications. Smoking status, use of medications, serum total cholesterol, high-density lipoprotein cholesterol, triglycerides, blood glucose, serum creatinine, and urine ACR were recorded from patient records. Current smoking was defined as having smoked the last cigarette within 1 week of the blood pressure and arterial stiffness measurements. All non-invasive measurements, including blood pressure, pulse wave analysis, and arterial stiffness measures were made by one trained investigator (J.L.Z). The study protocol was reviewed and approved by the Ethics Committee of Ruijin Hospital, Shanghai Jiaotong University School of Medicine. All patients provided written informed consent. 
Zuo et al.: Association of Haemodynamic Indices of Central and Peripheral Pressure with Subclinical Target Organ Damage

Clinical and Haemodynamic Measurements

Brachial and Pulse Wave Analysis Measurement

Radial pressure waveforms were obtained with applanation tonometry using a high-fidelity SPT-304 micromanometer (Millar Instruments, Houston, TX, USA) interfaced with a laptop computer, and central AP waveforms were derived using the SphygmoCor software, version 8.0 (AtCor Medical). Radial waveforms were calibrated with the average of the peripheral (brachial) SBP and DBP measured 3 times at the left brachial artery with a validated Omron 705CP oscillometric device (Omron, Kyoto, Japan). Peripheral PP (pPP) was calculated as the difference between SBP and DBP. Recordings were discarded when the systolic or diastolic variability of consecutive waveforms exceeded $5 \%$ or when the amplitude of the pulse wave signal was $<80 \mathrm{mV}$. All recordings met the manufacturer's quality control standards integrated into the software package. Augmentation index (AIx) was computed from the augmentation of AP as a percentage of central aortic PP (cPP). Systolic pressure amplification was defined as the difference between peripheral and central SBP. The amplification of PP from the aorta to the radial artery was defined as $\mathrm{pPP} / \mathrm{cPP}$. Form factor (FF) was defined as the ratio of mean pressure (MP) minus DBP to PP, i.e. FF = (MP - DBP)/PP [12]. FF was calculated for both radial (FFr) and central aortic (FFc) waveforms.

TOD Indices

TOD was defined as the presence of increased arterial stiffness, carotid IMT above normal values and/ or renal abnormalities as assessed by urine ACR above normal values.

Carotid-Femoral Pulse Wave Velocity. cfPWV was calculated as the ratio of the direct distance between the carotid and femoral sites of measurements and pulse transit time. The travel time between the two arterial sites was calculated as the difference between the R-wave of the electrocardiogram and the diastolic foot at the respective sites averaged over 10 consecutive heart beats. Following the measurement of office blood pressure, the carotid and femoral arterial waveforms at the patient's right side were recorded by applanation tonometry sequentially a short time apart by a trained investigator (J.L.Z.). Patients fasted overnight, and no caffeine beverage or smoking was allowed within $3 \mathrm{~h}$ of the measurement. A single high-fidelity applanation tonometer (Complior SPIV, France) was used. Increased arterial stiffness as subclinical TOD was defined as $\mathrm{cfPWV}>12 \mathrm{~m} / \mathrm{s}$.

Intima-Media Thickness. The IMT of carotid arteries was examined bilaterally using high-resolution echocardiography Doppler ultrasound (HD11EX Ultrasound; Philips Medical Systems, Andover, MA, USA) with a broadband linear array transducer (multiple frequency: 4-12 MHz). Three recordings were taken from both left and right carotid arteries, and the mean value was calculated for each side. Carotid plaque was defined as IMT $>1.3 \mathrm{~mm}$. Carotid abnormalities were diagnosed as IMT $\geq 0.9 \mathrm{~mm}$ and/or the presence of carotid plaque.

Renal Abnormalities: ACR. Urinary ACR was used to screen patients with urinary albuminuria. ACR values have been shown to identify kidney disease that occurs as a complication with hypertension. Abnormal albuminuria was defined as a urine ACR $>3.5 \mathrm{mg} / \mathrm{mmol}$ in females and $>2.5 \mathrm{mg} / \mathrm{mmol}$ in males [1].

\section{Statistical Analysis}

All analyses were performed using SPSS 24.0 for Windows (SPSS Inc., Chicago, IL, USA). A 2-sided $p<$ 0.05 was considered statistically significant. Continuous variables are expressed as mean $\pm \mathrm{SD}$. Independent differences in frequency were tested by $\chi^{2}$ analysis in univariate analysis between subclinical TOD and arterial stiffness.

Partial correlation (adjusted for age, sex, smoking, body mass index, use of antihypertensive drugs, lowdensity lipoprotein cholesterol, fasting blood glucose, and heart rate) was used to assess the relations between brachial, central aortic blood pressure and TOD. William's $\mathrm{T}_{2}$ statistic was used to test the differences in the correlations of central and peripheral haemodynamic indices (cPP vs. pPP, FFc vs. FFc) with TOD indices. Differences in the haemodynamic indices of central aortic and peripheral pressure between patients with or without TOD were estimated by means of univariate analyses. The association of haemodynamic indices with cfPWV was assessed by means of linear regression. Association between individual central and peripheral haemodynamic indices with the presence of TOD was assessed by means of logistic regression analysis with odds ratio (OR) calculation adjusted for age, sex, smoking, body mass index, use of antihypertensive drugs, fasting glucose, LDL cholesterol, heart rate, and DBP. 
Zuo et al.: Association of Haemodynamic Indices of Central and Peripheral Pressure with Subclinical Target Organ Damage

Table 1. General characteristics of the study population

\begin{tabular}{|c|c|c|c|c|}
\hline & Total & Male & Female & $p$ value \\
\hline Patients, $n$ & 770 & 473 & 297 & \\
\hline Age, years & $60 \pm 10$ & $60 \pm 11$ & $61 \pm 9$ & 0.85 \\
\hline BMI & $25.1 \pm 3.1$ & $25.3 \pm 3.2$ & $24.8 \pm 3.1$ & 0.03 \\
\hline Smoking, $n(\%)$ & $209(27)$ & $209(44)$ & 0 & $<0.01$ \\
\hline $\mathrm{FBG}, \mathrm{mmol} / \mathrm{L}$ & $5.8 \pm 1.5$ & $5.8 \pm 1.5$ & $5.8 \pm 1.6$ & 0.98 \\
\hline LDL cholesterol, mmol/L & $2.9 \pm 0.9$ & $2.8 \pm 0.8$ & $3.0 \pm 0.9$ & $<0.01$ \\
\hline $\log$ ACR & $0.37 \pm 0.53$ & $0.36 \pm 0.55$ & $0.38 \pm 0.48$ & 0.63 \\
\hline IMT, mm & $0.75 \pm 0.17$ & $0.82 \pm 0.17$ & $0.81 \pm 0.17$ & 0.57 \\
\hline $\mathrm{cfPWV}, \mathrm{m} / \mathrm{s}$ & $12.6 \pm 3.0$ & $12.8 \pm 3.1$ & $12.4 \pm 2.9$ & 0.11 \\
\hline Heart rate, bpm & $71 \pm 10$ & $71 \pm 11$ & $70 \pm 10$ & 0.25 \\
\hline \multicolumn{5}{|l|}{ Peripheral blood pressure } \\
\hline $\mathrm{SBP}, \mathrm{mm} \mathrm{Hg}$ & $142 \pm 20$ & $142 \pm 20$ & $142 \pm 20$ & 0.6 \\
\hline DBP, mm Hg & $82 \pm 11$ & $83 \pm 11$ & $80 \pm 11$ & $<0.01$ \\
\hline $\mathrm{PP}, \mathrm{mm} \mathrm{Hg}$ & $60 \pm 15$ & $60 \pm 16$ & $62 \pm 15$ & 0.07 \\
\hline Form factor, $\%$ & $33.3 \pm 0.5$ & $33.4 \pm 0.54$ & $33.2 \pm 0.52$ & 0.14 \\
\hline \multicolumn{5}{|l|}{ Central blood pressure } \\
\hline $\mathrm{SBP}, \mathrm{mm} \mathrm{Hg}$ & $121 \pm 19$ & $129 \pm 20$ & $132 \pm 19$ & 0.04 \\
\hline DBP, mm Hg & $83 \pm 11$ & $84 \pm 12$ & $82 \pm 11$ & $<0.01$ \\
\hline $\mathrm{PP}, \mathrm{mm} \mathrm{Hg}$ & $47 \pm 14$ & $40 \pm 13$ & $50 \pm 13$ & $<0.01$ \\
\hline $\mathrm{AP}, \mathrm{mm} \mathrm{Hg}$ & $13 \pm 8$ & $11 \pm 7$ & $17 \pm 7$ & $<0.01$ \\
\hline AIx & $26.2 \pm 10.9$ & $22.6 \pm 10.4$ & $32.1 \pm 8.9$ & $<0.01$ \\
\hline AIx@HR75, \% & $24.2 \pm 9.5$ & $20.9 \pm 9.1$ & $29.6 \pm 7.4$ & $<0.01$ \\
\hline Form factor, $\%$ & $43.8 \pm 4.1$ & $44.1 \pm 4.5$ & $43.4 \pm 3.3$ & 0.02 \\
\hline pSBP-cSBP, mm Hg & $25 \pm 5$ & $14 \pm 6$ & $10 \pm 5$ & $<0.01$ \\
\hline $\mathrm{pPP} / \mathrm{cPP}$ & $1.31 \pm 0.17$ & $1.36 \pm 0.17$ & $1.24 \pm 0.13$ & $<0.01$ \\
\hline
\end{tabular}

Data are mean \pm SD or as stated. $p$ value: independent $t$ test analysis of variance for numeric variables and $\chi^{2}$ test for categorical variables. cfPWV, carotid femoral pulse wave velocity; pPP, peripheral pulse pressure; CPP, central pulse pressure; AP, central augmentation pressure; AIx, central augmentation index; AIx@HR75, AIx adjusted to heart rate of $75 \mathrm{bpm}$.

Table 2. Partial correlation coefficients among variables adjusted by confounding factors

\begin{tabular}{|c|c|c|c|c|c|c|c|c|c|}
\hline & \multicolumn{3}{|l|}{ cf PWV } & \multicolumn{3}{|l|}{$\log A C R$} & \multicolumn{3}{|l|}{ IMT } \\
\hline & total & male & female & total & male & female & total & male & female \\
\hline $\mathrm{pPP}$ & $0.40^{* *}$ & $0.40^{* *}$ & $0.40^{* *}$ & $0.24^{* *}$ & $0.26^{* *}$ & $0.16^{*}$ & $0.14^{* *}$ & $0.12^{*}$ & $0.16^{*}$ \\
\hline $\mathrm{cPP}$ & $0.41^{* *}$ & $0.43^{* *}$ & $0.38^{* *}$ & $0.27^{* *}$ & $0.30^{* *}$ & $0.17^{*}$ & $0.15^{* *}$ & $0.11^{*}$ & $0.19^{* *}$ \\
\hline pSBP-cSBP & $0.11^{* *}$ & 0.04 & $0.23^{* *}$ & 0.008 & 0.001 & 0.001 & 0.06 & 0.06 & -0.02 \\
\hline $\mathrm{AP}$ & $0.28^{* *}$ & $0.33^{* *}$ & $0.21^{* *}$ & $0.20^{* *}$ & $0.23^{* *}$ & 0.13 & $0.10^{*}$ & 0.07 & 0.12 \\
\hline AIx & $0.14^{* *}$ & $0.23^{* *}$ & $-0.17^{*}$ & $0.13^{* *}$ & $0.16^{* *}$ & 0.07 & 0.07 & 0.09 & 0.03 \\
\hline AIx@HR75 & $0.16^{* *}$ & $0.25^{* *}$ & -0.04 & $0.11^{*}$ & $012^{*}$ & 0.07 & 0.04 & 0.06 & 0.02 \\
\hline $\mathrm{pPP} / \mathrm{cPP}$ & $-0.15^{* *}$ & $-0.22^{* *}$ & 0.001 & $-0.15^{*}$ & $-0.17^{* *}$ & -0.09 & -0.07 & -0.05 & -0.08 \\
\hline FFr & -0.05 & 0.001 & -0.12 & -0.01 & -0.02 & -0.07 & -0.01 & -0.007 & -0.04 \\
\hline $\mathrm{FFc}$ & $-0.10^{*}$ & -0.08 & -0.13 & $-0.13^{* *}$ & $-0.19^{* *}$ & 0.08 & $-0.10 *$ & -0.10 & 0.08 \\
\hline
\end{tabular}

After adjusting for age, sex, smoking, and body mass index, use of antihypertensive drugs, LDL cholesterol, fasting blood glucose, and heart rate. Significance ${ }^{*} p<0.05,{ }^{* *} p<0.01$. PWV, carotid femoral pulse wave velocity; $\mathrm{PPP}$, peripheral pulse pressure; $\mathrm{cPP}$, central aortic pulse pressure; $\mathrm{AP}$, central aortic augmentation pressure; AIx, central aortic augmentation index; AIX@HR75 (\%), AIx corrected to heart rate of 75 beats/ min; FFr, form factor for radial pressure waveform; FFc, form factor for central aortic waveform. 
Zuo et al.: Association of Haemodynamic Indices of Central and Peripheral Pressure with Subclinical Target Organ Damage

Table 3. Determinants of pulse wave velocity

\begin{tabular}{lccccc}
\hline Variable & $\mathrm{B}$ & $\mathrm{SE}$ & $\beta$ & $p$ value & $R^{2}$ \\
\hline pPP & 0.07 & 0.006 & 0.356 & $<0.001$ & 0.42 \\
cPP & 0.08 & 0.008 & 0.372 & $<0.001$ & 0.42 \\
AP & 0.096 & 0.016 & 0.25 & $<0.001$ & 0.36 \\
pSBP-cSBP & 0.094 & 0.017 & 0.18 & $<0.001$ & 0.34 \\
FFc & -6.91 & 2.65 & -0.095 & $<0.01$ & 0.33 \\
\hline Additional classified by & age ( $\geq 60$ vs. $<60$ years) & & & \\
AIx & 0.038 & 0.013 & 0.13 & $<0.001$ & 0.24 \\
AIx@HR75 & 0.038 & 0.014 & 0.12 & $<0.01$ & 0.24 \\
pPP/cPP & -3.19 & 0.834 & -0.17 & $<0.001$ & 0.25 \\
\hline
\end{tabular}

After adjusting for age, sex, smoking, and body mass index, use of antihypertensive drugs, LDL cholesterol and glucose, heart rate, and diastolic blood pressure. pPP, peripheral pulse pressure; cPP, central pulse pressure; AP, central augmentation pressure; pSBP, peripheral SBP; cSBP, central SBP; AIx, central augmentation index; AIX@HR75 (\%), AIx corrected to heart rate of 75 beats/min; FFc, form factor for central aortic waveform.

\section{Results}

For the whole study cohort ( $n=770), 194(25 \%)$ and $446(58 \%)$ patients presented abnormality of ACR and carotid artery wall, respectively. The clinical characteristics and haemodynamic indices of the study cohort are shown in Table 1. All augmented pressure indices (AP, AIx, and AIx adjusted to heart rate at 75 bpm, AIx@HR75) as well as pPP and cPP were greater in females than in males. However, peripheral and central DBP, systolic pressure amplification, PP amplification, and FFc were higher in males. There were no differences in cfPWV and FFr between males and females. When adjusted for age, sex, smoking, body mass index, use of antihypertensive drugs, low-density lipoprotein cholesterol, fasting blood glucose, heart rate and DBP, there were no differences in pPP between males and females.

Table 2 summarizes the partial correlations between haemodynamic indices and TOD indices adjusted for confounding factors. All haemodynamic indices showed a significant positive correlation with cfPWV, except for FFr. cPP and pPP were positively correlated with ACR. There was a significant negative correlation of PP amplification with cfPWV and ACR, respectively, but not with carotid IMT. Both pPP and cPP correlated positively with carotid IMT. Overall, there were different strengths of correlation between males and females. In general, cPP had higher correlations with TOD indices than pPP, with the strengths of the correlations reaching statistical significance for ACR ( $p=0.031$ for difference). In males, the strength of the correlation between cPP and cfPWV was greater than that between pPP and cfPWV, with the difference being slightly below statistical significance ( $p=0.072$ ).

Using cfPWV as the independent continuous variable in multiple linear regression, cPP, pPP, and AP were all positively associated with cfPWV, whereas FFc was negatively associated with cfPWV after adjusting for confounding factors such as age, sex, smoking, body mass index, use of antihypertensive drugs, low-density lipoprotein cholesterol, fasting blood glucose and heart rate (Table 3). For age >60 years, AIx@ 0 HR75 and PP amplification showed stronger positive associations with cfPWV than that in those younger than 60 years of age.

For patients with cfPWV higher than $12 \mathrm{~m} / \mathrm{s}$, albuminuria or carotid abnormalities showed higher pPP, cPP, AP, and AIx as compared with those without these conditions (Fig. 1). After adjusting for confounding factors, each SD increase in pPP was associated with 2.7, 1.2, 

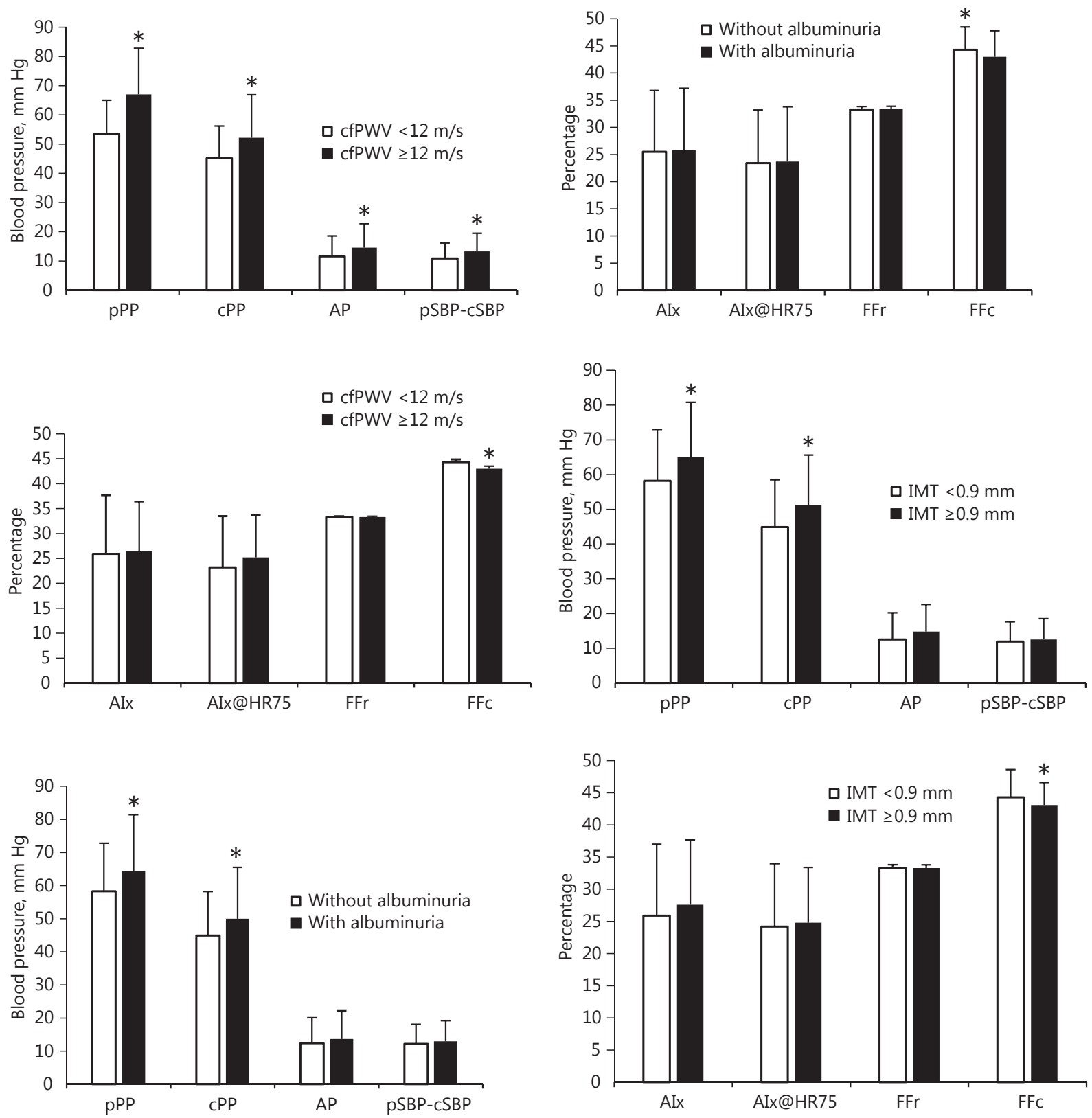

Fig. 1. Pressure indices for categories with (dark bars) and without (light bars) TOD. Patients with cfPWV $>12 \mathrm{~m} / \mathrm{s}$, albuminuria, or carotid IMT showed higher pPP, cPP, AP, and AIx.

and 1.5 times higher risk of increased arterial stiffness (cfPWV $\geq 12 \mathrm{~m} / \mathrm{s}$ ), albuminuria (ACR $>3.5 \mathrm{mg} / \mathrm{mmol}$ in females or $>2.5 \mathrm{mg} / \mathrm{mmol}$ in males) or carotid abnormalities (IMT $>0.9$ $\mathrm{mm}$ ), respectively (Table 4). Each SD increase in cPP entailed a 2.9 times higher risk of increased arterial stiffness, a 1.4 times higher risk of the occurrence of albuminuria and 1.5 times higher risk of increased carotid IMT. Each SD increase in central aortic AIx and systolic pressure amplification was associated with 0.7 times higher risk of occurrence of increased 
Zuo et al.: Association of Haemodynamic Indices of Central and Peripheral Pressure with Subclinical Target Organ Damage

Table 4. Associations of central aortic and peripheral haemodynamic indices with TOD indices as determined by separate multi-logistic regression models

\begin{tabular}{|c|c|c|c|c|c|c|}
\hline Variable & B & SE & $\chi^{2}$ & $\mathrm{OR}$ & $95 \% \mathrm{CI}$ & $p$ value \\
\hline \multicolumn{7}{|l|}{$\mathrm{cfPWV}>12 \mathrm{~m} / \mathrm{s}$} \\
\hline $\mathrm{pPP}, \mathrm{SD}$ & 0.981 & 0.124 & 62.6 & 2.666 & $2.09-3.4$ & $<0.001$ \\
\hline $\mathrm{cPP}, \mathrm{SD}$ & 1.05 & 0.138 & 58.3 & 2.857 & $2.182-3.741$ & $<0.001$ \\
\hline $\mathrm{AP}, \mathrm{SD}$ & 0.524 & 0.124 & 17.7 & 1.688 & $1.323-2.154$ & $<0.001$ \\
\hline pSBP-cSBP & 0.077 & 0.016 & 22.081 & 1.080 & $1.046-1.116$ & $<0.001$ \\
\hline $\mathrm{FFc}, \mathrm{SD}$ & -0.352 & 0.123 & 8.25 & 0.703 & $0.553-0.894$ & 0.004 \\
\hline \multicolumn{7}{|c|}{ Additional classified by age ( $\geq 60$ years) } \\
\hline $\mathrm{pPP} / \mathrm{cPP}$ & -2.551 & 1.055 & 5.853 & 0.078 & $0.1-0.616$ & 0.016 \\
\hline (Male) AIx@HR75,SD & 0.431 & 0.195 & 4.897 & 1.539 & $1.051-2.255$ & 0.027 \\
\hline \multicolumn{7}{|c|}{ ACR $>3.5 \mathrm{mg} / \mathrm{mmol}$ in females and $>2.5 \mathrm{mg} / \mathrm{mmol}$ in males } \\
\hline $\mathrm{pPP}, \mathrm{SD}$ & 0.361 & 0.097 & 4.23 & 1.155 & $1.026-1.301$ & 0.017 \\
\hline $\mathrm{cPP}, \mathrm{SD}$ & 0.325 & 0.094 & 11.85 & 1.384 & $1.15-1.664$ & 0.001 \\
\hline $\mathrm{FFc}, \mathrm{SD}$ & -0.251 & 0.097 & 6.650 & 0.778 & $0.643-0.942$ & 0.01 \\
\hline \multicolumn{7}{|l|}{$\mathrm{IMT}>0.9 \mathrm{~mm}$} \\
\hline $\mathrm{pPP}, \mathrm{SD}$ & 0.381 & 0.091 & 17.6 & 1.464 & $1.225-1.748$ & $<0.001$ \\
\hline cPP, SD & 0.428 & 0.094 & 20.544 & 1.534 & $1.275-1.846$ & $<0.001$ \\
\hline $\mathrm{FFc}, \mathrm{SD}$ & -0.294 & 0.094 & 9.728 & 0.745 & $0.619-0.896$ & 0.002 \\
\hline
\end{tabular}

After adjusting for age, sex, smoking, and body mass index, use of antihypertensive drugs, LDL cholesterol and glucose, heart rate, and diastolic blood pressure. cfPWV, carotid femoral pulse wave velocity; pPP, peripheral pulse pressure; $\mathrm{CPP}$, central pulse pressure; AP, central augmentation pressure; pSBP, peripheral SBP; cSBP, central SBP; AIx, central augmentation index; AIX@HR75 (\%), AIx corrected to heart rate; FFc, form factor for central. (Male) AIx@HR75, further classified by sex.

arterial stiffness. For FFc, each SD decrease was associated with a decreased OR of 0.7 for cfPWV over $12 \mathrm{~m} / \mathrm{s}, 0.8$ for albuminuria, and of 0.8 for carotid IMT, respectively. For age $>60$ years, decreased PP amplification was associated with an increase in risk of cfPWV over 12 m/s. For males over the age of 60 years, each SD increase in AIx@HR75 was associated with 1.5 times increase in risk of cfPWV over $12 \mathrm{~m} / \mathrm{s}$. When cPP and pPP were forced into the same logistic regression model, cPP remained a significant determinant $(\mathrm{OR}=3.1$ for each $\mathrm{SD}$ increase in $c P P, p<0.001$ ) of increased aortic stiffness for age $\geq 60$ years, but $p P P$ was not.

\section{Discussion}

The main findings of this study are that cPP, pPP, and FFc are related to TOD as assessed by increased cfPWV, urinary ACR, and carotid IMT in a cohort of patients with essential hypertension. Haemodynamic indices of central and peripheral pressure are independent risk factors for arterial stiffness as measured by cfPWV. As indicated by partial correlation coefficients ( $\mathrm{r}$ ), multiple linear regression coefficients and OR, this study has demonstrated that cPP has a more robust correlation with TOD than pPP. In addition, we confirmed that cPP is more strongly associated with age than pPP. Consequently, for age $\geq 60$ years, cPP provides additional information beyond $\mathrm{pPP}$ when adjusted for confounding factors.

$\mathrm{PP}$ was regarded as one of the factors influencing prognosis of hypertension in the elderly by the ESH/ESC guidelines for the management of hypertension [1]. PP is a significant determinant of subclinical TOD. Although conventional office peripheral blood pressure measurement currently remains the "gold standard" for screening, diagnosis and management 
of hypertension, it is generally accepted that central AP provides significant adjunct information. Due to the variable superposition of incident and reflected pressure waves along the arterial tree, central aortic blood pressure is generally not identical to brachial blood pressure.

Recent studies show an increasing interest in the use of central AP measured by noninvasive methods to assess the association with TOD [13]. To date there have been a number of studies in large cohorts assessing the relationship between cPP versus pPP with TOD. cfPWV has been shown to be related to TOD in asymptomatic hypertensive patients, [14]. In a recent meta-analysis by Kollias et al. [13], cPP was shown to be more closely associated with indices of subclinical TOD, quantified as cfPWV, left ventricular mass index, carotid IMT, and urine ACR, when compared with peripheral PP [13]. The pooled correlations between cPP and pPP with cfPWV ( $r=0.41$ and 0.35 for cPP and pPP, respectively) were similar to the findings of the present study ( $r=0.41$ and 0.40 for cPP and pPP, respectively), but the correlation coefficients were higher for ACR and lower for carotid IMT in the present study. Some studies show that urinary albumin excretion assessed by 24 -h urine protein or ACR $[3,15]$ have similar correlation with central and peripheral pressure. In our study, we found that cPP was more closely correlated with urinary albumin excretion assessed by ACR than pPP. This observation has been previously explained in terms of the changes of PP along the arterial tree including at the level of renal arteries [16]. cPP can be considered to be integrated in the haemodynamic function of the aorta and kidneys and may have a more significant influence than brachial PP in the determination of CV risk. Vice versa, central aortic blood pressure can be more closely related to the pressure in the abdominal AP (at the level of kidneys), than peripheral blood pressure. Furthermore, our study suggests that some patients with higher $\mathrm{cfPWV}, \mathrm{ACR}$, or carotid IMT will have a greater benefit by a reduction of $\mathrm{cPP}$ compared to a similar reduction in $\mathrm{pPP}$, and so targeting reduction of cPP may be a more effective therapeutic strategy.

An earlier study [15] in middle-aged hypertensive patients showed that cPP was more closely correlated with cfPWV and carotid IMT than pPP, with equal associations with ACR. When adjusted for relevant co-factors, cPP did not provide additional information beyond pPP. In our study, we obtained similar unadjusted results, showing cPP to be more closely correlated with cfPWV and carotid IMT compared to pPP, as well as ACR. However, in our cohort, these associations remained following adjustment for confounding factors, showing that cPP provides potential additional information beyond $\mathrm{pPP}$. The reason for these different results is mainly in the difference in adjusting for confounding factors. The fact that age [17, 18], antihypertensive drugs [19], body mass index or height [20] can influence the amplification of systolic pressure between central and upper limb arteries can play an important role in the adjusted factors. For example, antihypertensive therapy can have different effects on central and peripheral systolic pressure, influencing TOD, reducing left ventricular hypertrophy (LVH), cfPWV, carotid IMT and ACR $[19,21]$. Another study found that central aortic blood pressure, even when monitored for $24 \mathrm{~h}$ with an oscillometric device for pulse wave velocity [22], had similar associations with TOD to peripheral blood pressure [23]. However, the aforementioned study did not measure cfPWV but calculated it from a single point measurement of the pulse wave in the brachial cuff [23]. Although this measurement is not yet used to for clinical assessment of TOD, some studies are starting to show significant differences in aortic PWV between groups measured with this technique [24].

In normotensive younger subjects, the peripheral (pSBP) to central (cSBP) SBP discrepancy is generally higher than that in older subjects $[25,26]$. The discrepancy between peripheral and central SBP mainly depends on arterial stiffness and wave reflection. However, isolated systolic hypertension (ISH) in youth is usually caused by high amplification of the pressure pulse in the arm from the aorta as quantified by pSBP-cSBP and pPP/cPP [17], whereas ISH in the elderly is mainly due to generalized stiffening of the central aorta and large 
conduit arteries [18]. Therefore, haemodynamic indices of central AP can be classified by different age and gender. In our study, haemodynamic indices (PP, AIx, and AIx@HR75) decreased in males, and led to greater difference between peripheral and central aortic SBP than in females. In addition, for AIx@HR75 and pPP/cPP, the prospective values of arterial stiffness are limited by age and gender respectively. FF was introduced to provide a simple numerical characterization of wave shape [12]. Generally, a low FF (e.g., 30\%) corresponds to a sharply peaked wave, whereas a high FF (above 40\%) is associated with a blunted pressure wave [27]. In this study, FF for the central AP pulse has been found to be more closely related to the three types of TOD indices (arterial stiffness, renal abnormalities, and increased carotid IMT). As a wave shape measurement, further studies are required before recommending FF as a routine parameter of central aortic blood pressure for clinical application.

\section{Limitations}

The findings of this study are limited by several factors. First, the cross-sectional design of the study allows only descriptive associations instead of predictive value of central or peripheral PP in the progression of TOD. Second, there is lack of data on LVH as a marker of subclinical TOD. Since aortic blood pressure is the direct afterload of the left ventricle [28], theoretically it has a stronger relationship with LVH compared to PP [29]. However, this study did not use cardiac ultrasonic means to evaluate LVH. For non-invasive assessment of cfPWV, estimation of pulse wave travel distance is critical. The best measurements were found for the method of subtracting the carotid-suprasternal notch distance from the suprasternal notchfemoral distance [30, 31]. In our study, the direct distance between the carotid and femoral sites was used, and this may have led to an overestimation of the values for cfPWV. However, this is not a major limitation; while the subtraction method is associated with a cut-off threshold of $10 \mathrm{~m} / \mathrm{s}$ [32], the threshold for the total direct distance method is $12 \mathrm{~m} / \mathrm{s}$ [33, 34], which was the value used in this study. Finally, it is acknowledged that non-invasive estimations of central APs are dependent on the accuracy of the calibration method [35]. In the present study, cuffmeasured non-invasive brachial SBP and DBP were used to calibrate the non-invasive radial waveform used for deriving central APs. Although blood pressure as measured by a brachial cuff has been shown to have variable accuracy [36] and thus leads to a flow-on effect of variable accuracy of central aortic blood pressure estimation, the ratio of central to peripheral pressures remains the same whether measured invasively or non-invasively.

\section{Conclusion}

cPP, pPP, and FFc have been found to be more closely related to TOD assessed as increased cfPWV, urinary ACR, and carotid IMT. Haemodynamic indices of central and peripheral pressure are independent risk factors (determinants) of arterial stiffness as measured by cfPWV. cPP predicting TOD in hypertension may be superior than pPP in the elderly. For age $\geq 60$ years, cPP provides additional information beyond pPP. Haemodynamic indices of central pressure as biomarkers of subclinical TOD need to be validated by further prospective studies.

\section{Acknowledgements}

We gratefully acknowledge the invaluable assistance of the physicians of the Department of Hypertension, Ruijin Hospital, Shanghai Jiaotong University School of Medicine. The study would not have been possible without their support. 
Zuo et al.: Association of Haemodynamic Indices of Central and Peripheral Pressure with Subclinical Target Organ Damage

\section{Disclosure Statement}

None of the authors has a conflict of interest with regard to the data presented in this paper.

\section{Funding Sources}

Project Supported by the National Natural Science Foundation of China (Grant No. 81500190), and Shanghai Municipal Commission of Health and Family Planning (Grant No. 201740128).

\section{References}

1 Mancia G, Fagard R, Narkiewicz K, Redon J, Zanchetti A, Bohm M, et al: 2013 ESH/ESC Practice Guidelines for the Management of Arterial Hypertension. Blood Press 2014;23:3-16.

$\$ 2$ Avolio AP, Butlin M, Walsh A: Arterial blood pressure measurement and pulse wave analysis - their role in enhancing cardiovascular assessment. Physiol Meas 2010;31:R1-R47.

3 Oliveras A, García-Ortiz L, Segura J, Banegas JR, Martell-Claros N, Vigil L, et al: Association between urinary albumin excretion and both central and peripheral blood pressure in subjects with insulin resistance. J Hypertens 2013;31:103-108.

$\checkmark 4$ Roman MJ, Okin PM, Kizer JR, Lee ET, Howard BV, Devereux RB: Relations of central and brachial blood pressure to left ventricular hypertrophy and geometry: the Strong Heart Study. J Hypertens 2010;28:384388.

-5 Wang K-L, Cheng H-M, Chuang S-Y, Spurgeon HA, Ting C-T, Lakatta EG, et al: Central or peripheral systolic or pulse pressure: which best relates to target organs and future mortality? J Hypertens 2009;27:461-467.

6 Laurent S, Cockcroft J, Bortel L Van, Boutouyrie P, Giannattasio C, Hayoz D, et al: Expert consensus document on arterial stiffness: methodological issues and clinical applications. Eur Heart J 2006;27:2588-2605.

7 Safar ME, Blacher J, Pannier B, Guerin AP, Marchais SJ, Guyonvarc'h PM, London GM: Central pulse pressure and mortality in end-stage renal disease. Hypertension 2002;39:735-738.

8 Agabiti-Rosei E, Mancia G, O’Rourke MF, Roman MJ, Safar ME, Smulyan H, et al: Central blood pressure measurements and antihypertensive therapy: a consensus document. Hypertension 2007;50:154-160.

$\checkmark 9$ Vlachopoulos C, Aznaouridis K, O’Rourke MF, Safar ME, Baou K, Stefanadis C: Prediction of cardiovascular events and all-cause mortality with central haemodynamics: a systematic review and meta-analysis. Eur Heart J 2010;31:1865-1871.

10 Nilsson PM, Boutouyrie P, Laurent S: Vascular aging: a tale of EVA and ADAM in cardiovascular risk assessment and prevention. Hypertension 2009;54:3-10.

11 Zuo J, Chang G, Ge Q, Chu S: Association between arterial function and albuminuria to creatinine ratio, carotid artery intima-media thickness in essential hypertensive patients. Chin J Hypertens 2016;24:1047-1052.

$\checkmark 12$ Chemla D, Hébert J-L, Aptecar E, Mazoit J-X, Zamani K, Frank R, et al: Empirical estimates of mean aortic pressure: advantages, drawbacks and implications for pressure redundancy. Clin Sci (Lond) 2002;103:7-13.

-13 Kollias A, Lagou S, Zeniodi ME, Boubouchairopoulou N, Stergiou GS: Association of central versus brachial blood pressure with target-organ damage: systematic review and meta-analysis. Hypertension DOI: 10.1161/ HYPERTENSIONAHA.115.06066.

-14 Abellán-Huerta J, Prieto-Valiente L, Consuegra-Sánchez L, Montoro-García S, Salguero-Merino AB, MoralesLópez R, et al: Most advisable strategy in search of asymptomatic target organ damage in hypertensive patients. Hipertens Riesgo Vasc 2017;34:149-156.

15 Neisius U, Bilo G, Taurino C, McClure JD, Schneider MP, Kawecka-Jaszcz K, et al: Association of central and peripheral pulse pressure with intermediate cardiovascular phenoytpes. J Hypertens 2012;30:67-74.

-16 Temmar M, Jankowski P, Peltier M, Mouquet V, Dębicka-Dąbrowska D, Hamida F, et al: Intraaortic pulse pressure amplification in subjects at high coronary risk. Hypertension 2010;55:327-332.

17 Wilkinson IB, Franklin SS, Hall IR, Tyrrell S, Cockcroft JR: Pressure amplification explains why pulse pressure is unrelated to risk in young subjects. Hypertension 2001;38:1461-1466.

18 O’Rourke MF, Hashimoto J: Mechanical factors in arterial aging. A clinical perspective. J Am Coll Cardiol 2007; 50:1-13.

19 Williams B, Lacy PS, Thom SM, Cruickshank K, Stanton A, Collier D, et al: Differential impact of blood pressurelowering drugs on central aortic pressure and clinical outcomes: principal results of the Conduit Artery Function Evaluation (CAFE) study. Circulation 2006;113:1213-1225.

20 Pichler G, Martinez F, Vicente A, Solaz E, Calaforra O, Lurbe E, et al: Influence of obesity in central blood pressure. J Hypertens 2015;33:308-313.

21 Rinaldi ER, Yannoutsos A, Borghi C, Safar ME, Blacher J: Central hemodynamics for risk reduction strategies: additive value over and above brachial blood pressure. Curr Pharm Des 2015;21:730-736.

22 Wassertheurer S, Kropf J, Weber T, Giet M Van Der, Baulmann J, Ammer M: A new oscillometric method for pulse wave analysis: comparison with a common tonometric method. J Hum Hypertens 2010;24:498-504. 
23 de la Sierra A, Pareja J, Fernández-Llama P, Armario P, Yun S, Acosta E, et al: Twenty-four-hour central blood pressure is not better associated with hypertensive target organ damage than 24-h peripheral blood pressure. J Hypertens 2017;35:1.

-24 Luzardo L, Lujambio I, Sottolano M, da Rosa A, Thijs L, Noboa 0, et al: 24-h ambulatory recording of aortic pulse wave velocity and central systolic augmentation: a feasibility study. Hypertens Res 2012;35:980-987.

25 McEniery CM, Cockcroft JR, Roman MJ, Franklin SS, Wilkinson IB: Central blood pressure: current evidence and clinical importance. Eur Heart J 2014;35:1719-1725.

-26 McEniery CM, Yasmin, McDonnell B, Munnery M, Wallace SM, Rowe CV, et al: Central pressure: variability and impact of cardiovascular risk factors the Anglo-Cardiff Collaborative Trial II. Hypertension 2008;51:14761482 .

-27 Segers P, Rietzschel E, Heireman S, De Buyzere M, Gillebert T, Verdonck P, et al: Carotid tonometry versus synthesized aorta pressure waves for the estimation of central systolic blood pressure and augmentation index. Am J Hypertens 2005;18:1168-1173.

28 Nichols WW: Clinical measurement of arterial stiffness obtained from noninvasive pressure waveforms. Am J Hypertens 2005;18:3-10.

29 Sibiya MJ, Norton GR, Booysen HL, Tade G, Libhaber CD, Ballim I, et al: Aortic backward waves rather than stiffness account for independent associations between pulse pressure amplification and left ventricular mass in a young to middle-aged sample. J Am Soc Hypertens 2017;11:350-358.

30 Weber T, Ammer M, Rammer M, Adji A, O’Rourke MF, Wassertheurer S, et al: Noninvasive determination of carotid-femoral pulse wave velocity depends critically on assessment of travel distance: a comparison with invasive measurement. J Hypertens 2009;27:1624-1630.

31 Girerd N, Legedz L, Paget V, Rabilloud M, Milon H, Bricca G, et al: Outcome associations of carotid-femoral pulse wave velocity vary with different measurement methods. Am J Hypertens 2012;25:1264-1270.

-32 Van Bortel LM, Laurent S, Boutouyrie P, Chowienczyk P, Cruickshank JK, De Backer T, et al: Expert consensus document on the measurement of aortic stiffness in daily practice using carotid-femoral pulse wave velocity. J Hypertens 2012;30:445-448.

-33 Sehestedt T, Jeppesen J, Hansen TW, Rasmussen S, Wachtell K, Ibsen H, et al: Which markers of subclinical organ damage to measure in individuals with high normal blood pressure? J Hypertens 2009;27:1165-1171.

-34 Mancia G, De Backer G, Dominiczak A, Cifkova R, Fagard R, Germano G, et al: 2007 Guidelines for the Management of Arterial Hypertension: The Task Force for the Management of Arterial Hypertension of the European Society of Hypertension (ESH) and of the European Society of Cardiology (ESC). J Hypertens 2007;25:11051187.

35 Sharman JE, Avolio AP, Baulmann J, Benetos A, Blacher J, Blizzard CL, et al: Validation of non-invasive central blood pressure devices: ARTERY Society task force consensus statement on protocol standardization. Eur Heart J 2017;38:2805-2812.

36 Picone DS, Schultz MG, Otahal P, Aakhus S, Al-Jumaily AM, Black JA, et al: Accuracy of cuff-measured blood pressure. J Am Coll Cardiol 2017;70:572-586. 